\section{(6) OPEN ACCESS}

\title{
Identification of novel antiacetylated vimentin antibodies in patients with early inflammatory arthritis
}

\author{
Maria Juarez, ${ }_{1}$ Holger Bang, ${ }^{2}$ Friederike Hammar, ${ }_{1}$ Ulf Reimer, ${ }^{3}$ Bernard Dyke, ${ }_{1}^{1}$ \\ Iffita Sahbudin, ${ }^{1}$ Christopher D Buckley, ${ }^{1}$ Benjamin Fisher, ${ }^{1}$ Andrew Filer, ${ }^{1}$ \\ Karim Raza ${ }^{1,4}$
}

\begin{abstract}
Handling editor Tore K Kvien
- Additional material is published online only. To view please visit the journal online (http://dx.doi.org/10.1136/ annrheumdis-2014-206785).

${ }^{1}$ Research Laboratories, Centre for Translational Inflammation Research, University of Birmingham, Queen Elizabeth Hospital, Birmingham, UK ${ }^{2}$ Orgentec Diagnostika GmbH, Mainz, Germany ${ }^{3}$ JPT Peptide Technologies $\mathrm{GmbH}$, Berlin, Germany

${ }^{4}$ Department of Rheumatology, Sandwell and West Birmingham Hospitals NHS Trust, Birmingham, UK
\end{abstract}

\section{Correspondence to}

Professor Karim Raza, Research Laboratories, Centre for Translational Inflammation Research, University of Birmingham, Queen Elizabeth Hospital. Birmingham B15 2WB, UK:

K.Raza@bham.ac.uk

Received 13 October 2014 Revised 13 May 2015 Accepted 14 May 2015 Published Online First 9 July 2015

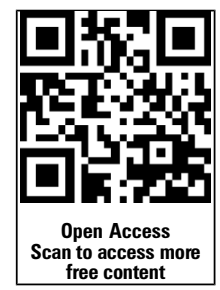

1099-1107.

\section{ABSTRACT}

Objective To investigate serum antibody reactivity against a panel of post-translationally modified vimentin peptides (PTMPs) in patients with early inflammatory arthritis.

Methods A panel of PTMPs was developed. Microtitre plates were coated with peptides derived from vimentin that were identical in length and composition except at one amino acid that was changed to introduce one of three post-translational modifications (PTMs) - either a citrullinated, carbamylated or acetylated residue. Sera of 268 treatment-naive patients with early inflammatory arthritis and symptoms $\leq 3$ months' duration were tested. Patients were assigned to one of three outcome categories at 18-month follow-up (rheumatoid arthritis (RA), persistent non-RA arthritis and resolving arthritis). Results Antibodies against citrullinated, carbamylated and acetylated vimentin peptides were detected in the sera of patients with early inflammatory arthritis. The proportion of patients seropositive for all antibody types was significantly higher in the RA group than in the other groups. Anti cyclic citrullinated peptide (CCP)positive patients with RA had higher numbers of peptides recognised and higher levels of antibodies against those peptides, representing a distinct profile compared with the other groups.

Conclusions We show for the first time that antibodies against acetylated vimentin are present in the sera of patients with early RA and confirm and extend previous observations regarding anticitrullinated and anticarbamylated antibodies.

\section{INTRODUCTION}

Rheumatoid arthritis (RA) is characterised by the presence of autoantibodies including those against citrullinated proteins (ACPAs) that are of diagnostic and prognostic relevance and have been implicated in disease pathogenesis. ${ }^{1-4}$ Testing for these antibodies is performed using the commercially available cyclic citrullinated peptide-2 (CCP-2) ELISA, whereby patients' sera are tested for reactivity against synthetic CCP that is not present in the RA joint. ${ }^{5}$ The sensitivities and specificities of this assay range between $60-80 \%$ and $88-95 \%$, respectively. ${ }^{5}$ Testing for ACPAs using other autoantigens such as citrullinated fibrinogen and mutated citrullinated vimentin $(\mathrm{MCV})$ is also possible although less frequently performed in routine clinical practice. ${ }^{6}$
MCV assays use an isoform of vimentin that is present in the RA joint, and their reported sensitivity and specificity are $82 \%$ and $98 \%$, respectively. ${ }^{7}$ Antibodies against another post-translational modification (PTM), anticarbamylated protein antibodies, have recently been identified in the sera of patients with RA where they have a prognostic role. ${ }^{8}$ It is thus possible that antibodies against further PTMPs are present in the sera of patients with RA.

PTMs are common biological processes generating structural and functional diversity in an otherwise limited proteome. ${ }^{9}$ Acetylation is a reversible enzymatic process where acetyl groups are added to free amines of lysine residues. Citrullination is an enzymatic process, catalysed by peptidylarginine deiminases (PADIs) that deiminate arginine to citrulline. In contrast, carbamylation is a non-enzymatic process that generates homocitrulline when cyanate ions react with amino groups of lysines and arginines. Citrulline and homocitrulline are structurally very similar, differing only by one carbon atom that makes the homocitrulline side chain longer (see online supplementary figure S1A). ${ }^{10}$ Acetylated lysine is identical to homocitrulline except at the side chain terminal amine, which is replaced by a methyl moiety.

PTMPs act as autoantigens in a variety of diseases. ${ }^{11}$ Citrullination occurs during several biological processes and during inflammation. In RA, citrullination takes place in the lungs, periodontal tissue and synovium. Citrullinated proteins are present in bronchoalveolar lavage fluid, cells and mucosa of smokers where they associate with higher PADI2 levels. Furthermore, parenchymal changes and citrullinated proteins have been identified in the lungs of patients with early ACPA-positive RA. ${ }^{12} 13$ This, together with extensive data linking smoking to a higher susceptibility of ACPA-positive RA, has led to the hypothesis that long-term exposure to cigarette smoke may induce citrullination of self-antigens leading to ACPA production in susceptible individuals. ${ }^{14}$

Acetylation of lysine residues in histone proteins plays a key role in nuclear transcription regulation. ${ }^{15}$ Acetylation of cytoplasmic proteins regulates enzymatic functions and metabolic pathways ${ }^{16}{ }^{17}$ and is a mechanism through which the microbiome can affect its host. ${ }^{18}$ To date, there have been no studies of immunity against acetylated peptides in RA. 
We investigated antibody reactivities against a panel of peptides bearing one of three PTM in a well-characterised early inflammatory arthritis patient cohort. We determined individual immune responses in relation to the patient's final clinical outcome to ascertain whether such analysis would help predict RA development in patients with early synovitis. Additionally, we sought to find new clues to potential disease triggers.

\section{PATIENTS AND METHODS \\ Patients}

Patients were recruited to the Birmingham early arthritis cohort. This is a cohort of disease-modifying antirheumatic drug (DMARD)-naive patients with clinically apparent synovitis of $\geq 1$ joint and inflammatory joint symptoms (morning stiffness and/or inflammatory joint pain and/or swelling) of $\leq 3$ months' duration. Patients are assigned to an outcome category at 18-month follow-up. Outcomes include persistent RA (fulfilling 1987 American College of Rheumatology (ACR) criteria), ${ }^{19}$ persistent non-RA arthritis and resolving arthritis. Resolving arthritis was defined as no clinically apparent joint swelling with no DMARD or steroid use in the previous three months. Classification of patients into outcome groups using the 2010 ACR European League Against Rheumatism classification criteria $^{20}$ was also performed. Data analyses according to these criteria are shown in online supplementary figures S3-S5 and tables S3 and S4. Sera of patients with symptom duration $>3$ months at presentation were also tested.

\section{Clinical and laboratory assessment}

Demographic and clinical parameters including age, gender, symptom duration, early morning stiffness duration, tender and swollen joint counts, erythrocyte sedimentation rate, $\mathrm{C}$ reactive protein, rheumatoid factor (RF) and anti-CCP status were recorded. Anti-CCP antibody levels were determined using a commercial anti-CCP2 assay (ELiA, Germany). Additional serum samples were stored at $-80^{\circ} \mathrm{C}$ for antibody panel analysis.

\section{Post-translationally modified peptides panel}

Antibodies against PTMP were determined by quantitative immunometric enzyme immunoassay (Orgentec Diagnostika $\mathrm{GmbH}$, Germany). A panel of PTMP was developed specifically for this purpose. Microtitre plates were precoated with streptavidin followed by addition of biotinylated peptides of identical length and composition that differed at one amino acid residue (JPT, Germany). The control peptide was a 12 amino acid sequence (GRVYATRSSAVR) derived from the vimentin protein used in the MCV assay. ${ }^{7}$ Position 7 (denoted by an $\mathrm{X}$ in online supplementary figure S1B) in this control peptide was changed to add the relevant modification. Thus, at position 7 the citrullinated vimentin peptide had citrulline, the carbamylated peptide had homocitrulline and the acetylated peptide had acetylated lysine. Further details of this assay are provided in online supplementary methods.

IgG and IgA antibodies against peptide panels were tested in healthy individuals $(n=160)$. Optical density values $>98$ th percentile of the healthy controls were considered positive for all antibodies. Online supplementary figure S2 shows the distribution of antibody levels in healthy individuals and patients.

\section{Immunoblotting and competitions studies}

Recombinant vimentin was citrullinated or carbamylated as previously described. ${ }^{8}$ For the acetylated isoform, albumin was conjugated with the acetylated vimentin peptide as recombinant vimentin is not stable after treatment with acetylation reagents. ${ }^{21}$ Unmodified and modified vimentin proteins were separated by SDS-PAGE, transferred to nitrocellulose and used for the immunoblotting experiments. Sera from patients with either triple $(n=4)$ or double $(n=5)$ reactivity were used to verify the specificity of the antibodies under denatured conditions. Sera were left untreated or preincubated with $10 \mu \mathrm{g} / \mathrm{mL}$ of control vimentin peptide or peptides with the relevant modification. Bound antibodies were detected by incubation with horseradish-peroxidase conjugated secondary antihuman IgG antibody (Dianova, Germany) and visualised with an ECL detection system (GE Healthcare, Germany).

Competition experiments using the immunoassay were also performed. Sera of triple reactive patients $(n=20)$ were sequentially preincubated with the native (arginine containing) vimentin peptide, each of the modified peptides or solvent from the peptide stock solution (used as untreated control). All experiments were run in triplicate. Resulting change in antibody binding was analysed with each microtiter plate (citrullinated, carbamylated and acetylated vimentin peptide). Data were calculated as percentage of residual binding relative to the solvent treated (non-peptide containing) control. Further details of this assay are provided in online supplementary methods.

\section{Statistical analysis}

All analyses were performed using PASW 20.0 (SPSS, USA). Data were expressed as percentages, mean $( \pm S D)$ or median (IQR) as appropriate. Comparisons were performed with $\chi^{2}$, analysis of variance and Kruskal-Wallis tests for categorical, parametric continuous and non-parametric continuous data, respectively. Post hoc analyses were performed with Sidack and Dunn's tests for parametric and non-parametric data, respectively. Binary logistic regression was used to calculate ORs.

\section{RESULTS}

Serum samples from 268 patients with early arthritis were available. Patients were categorised into three clinical outcome groups: RA $(n=101)$, persistent non-RA arthritis $(n=55)$ and resolving arthritis $(n=112)$. Demographic and clinical characteristics of patients are represented in table 1, where patients with RA have been subdivided into anti-CCP antibody-positive and antibody-negative groups. The individual diagnosis of patients in each outcome group is shown in online supplementary table S1.

\section{Antibody seropositivity}

The frequency of antibody seropositivity against different PTM vimentin peptides is represented in figure 1 . The proportion of patients with positive IgG antibody tests was significantly higher in the RA group compared with persistent non-RA and resolving arthritis groups for all modifications $(p<0.001$ for all). In contrast, the proportion of patients with a positive IgA antibody test was significantly higher in the RA group compared with the others only for the citrullinated peptide $(p=0.016)$.

\section{Full-length vimentin immunoblotting and competition studies}

To confirm that the results obtained with the immunometric assay were representative of the in vivo situation where the fulllength vimentin protein is present, sera from four patients with anti-CCP-positive RA that tested positive for IgG anti-CitVimentin, anti-CarVimentin and anti-AcVimentin antibodies (triple reactive) and five patients that tested positive for IgG anti-CitVimentin and anti-CarVimentin (double reactive) were analysed in full-length vimentin immunoblotting 
Table 1 Demographic, clinical and laboratory characteristics of patients in each outcome group

\begin{tabular}{|c|c|c|c|c|c|}
\hline & Anti-CCP-positive RA ( $n=48)$ & Anti-CCP-negative RA ( $n=53$ ) & Persistent non-RA ( $n=55)$ & Resolving arthritis $(n=112)$ & p-Value \\
\hline Gender, n (\%) & & & & & 0.545 \\
\hline Female & $30(62.5)$ & $26(49.1)$ & $30(54.5)$ & $58(51.8)$ & \\
\hline Age (years) & $53.1 \pm 13.4$ & $60.3 \pm 15.8$ & $49.4 \pm 19.4$ & $45.5 \pm 15.9$ & $<0.001$ \\
\hline Disease duration (days) & $57 \pm 22.7$ & $58.9 \pm 21.2$ & $54.1 \pm 22.1$ & $45.79 \pm 21.4$ & 0.001 \\
\hline CRP (mg/dL) & $18(6-40)$ & $13(6-41)$ & $22(8-38)$ & $8(0-20)$ & $<0.001$ \\
\hline ESR (mm/hour) & $29(20-53)$ & $23.5(13-56)$ & $25(12-40)$ & $14(7-29)$ & $<0.001$ \\
\hline DAS28 (CRP) & $4.3 \pm 1.3$ & $4.3 \pm 1.2$ & $3.8 \pm 1.1$ & $3.1 \pm 1.2$ & $<0.001$ \\
\hline DAS28 (ESR) & $4.8 \pm 1.1$ & $4.6 \pm 1.2$ & $4.1 \pm 1.3$ & $3.3 \pm 1.3$ & $<0.001$ \\
\hline Smoking, n (\%) & & & & & 0.01 \\
\hline Ever-smoker & $27 / 47(57.4)$ & $32 / 51(62.7)$ & 19/53 (35.8) & $42 / 103(40.8)$ & \\
\hline Never-smoker & $20 / 47(42.6)$ & 19/51 (37.3) & $34 / 53(64.2)$ & $61 / 103(59.2)$ & \\
\hline Anti-CCP positive, $n(\%)$ & $48(100)$ & $0(0)$ & $1(1.8)$ & $2(1.8)$ & $<0.001$ \\
\hline RF positive, $\mathrm{n}(\%)$ & $40 / 46(87.0)$ & $8 / 52(15.4)$ & $5 / 54(9.3)$ & $12 / 109(11.0)$ & $<0.001$ \\
\hline
\end{tabular}

experiments (figure 2A and online supplementary figure S6). Recombinant unmodified and modified vimentin proteins were probed with non-preincubated sera and sera preincubated with the native unmodified vimentin peptide or the modified peptides sequentially. Reactivity against the whole protein could be specifically blocked with a single dose of citrullinated, carbamylated or acetylated peptide, respectively. Probing with nonpreincubated sera and preincubation with the unmodified vimentin peptide had no blocking effect.
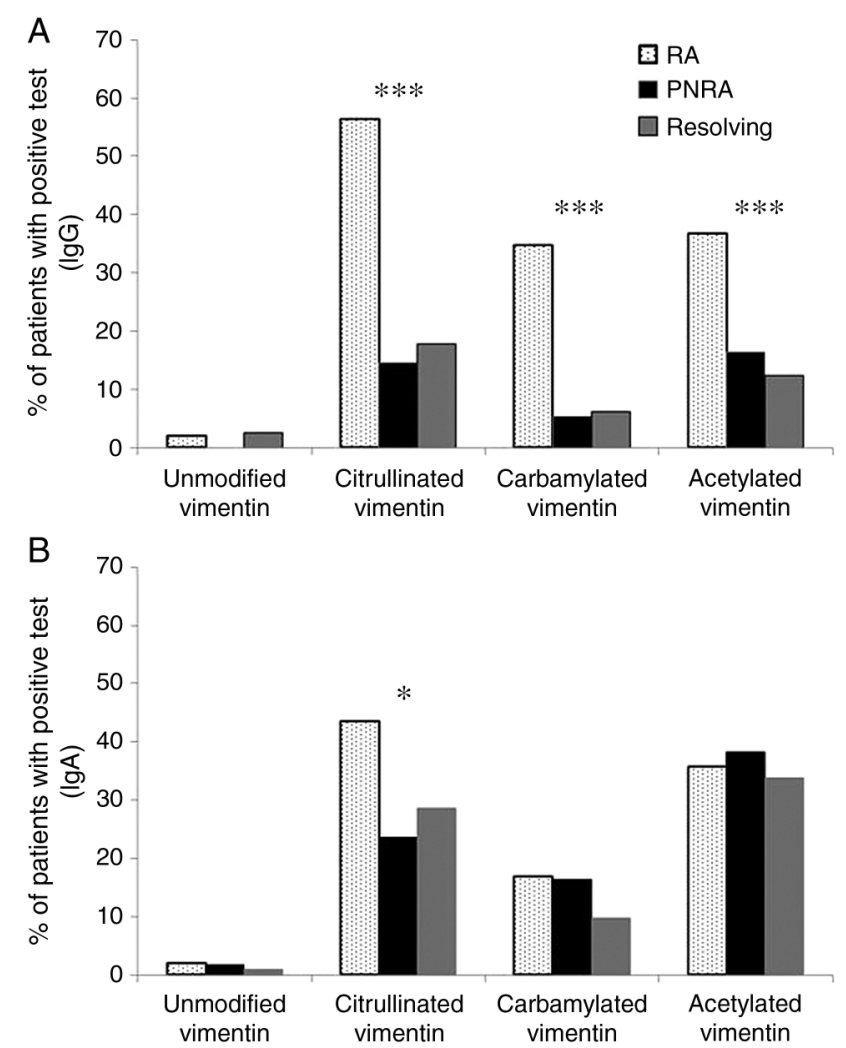

Figure 1 Frequency of seropositivity for $\lg G$ and $\lg A$ antibodies against different post-translationally modified peptides in rheumatoid arthritis (RA) (dotted bars), persistent non-RA (black bars) and resolving (grey bars) groups. (A) $\lg G$ antibody panel; (B) $\lg A$ antibody panel. ${ }^{*} \mathrm{p}<0.05,{ }^{* *} \mathrm{p} \leq 0.001$.
Next, we investigated whether reactivity in the immunoassay could be blocked by preincubation of patients' sera $(n=20)$ with the specific soluble peptides. Preincubation of patients' sera with the unmodified control vimentin peptide did not inhibit binding for any of the antibodies (figure 2B). Preincubation of sera with citrullinated, carbamylated and acetylated soluble peptides blocked antibody reactivity in a dose-dependent manner (figure 2C). To determine the degree of cross-reactivity between the different antibody populations, competition experiments were performed. Reactivity to anti-CitVimentin peptides was blocked by preincubation with the soluble citrullinated peptide as expected (70\% inhibition), with weak inhibition of binding by carbamylated $(23 \%)$ and acetylated $(17 \%)$ vimentin peptides (figure 2D). Similar specificity and low cross-reactivity was observed for the anti-AcVimentin antibodies. A degree of crossreactivity was observed for anti-CarVimentin antibodies where $32 \%$ of the binding was inhibited by preincubation with citrullinated soluble peptide.

\section{Sensitivity, specificity and predictive value of antibody panel}

To evaluate whether these antibodies would be a useful tool in predicting RA development, sensitivity, specificity, positive and negative predictive values for each antibody were calculated (table 2). Using fulfilment of 1987 ACR criteria as the gold standard, patients were grouped into two categories: RA and non-RA (the latter comprising resolving and persistent non-RA groups). Although some antibodies (eg, IgG anti-CarVimentin, IgG anti-AcVimentin) showed high specificities, their sensitivities were low. The best sensitivity and specificity value combination was demonstrated by anti-CCP antibodies. Sensitivities of the assays were greater when anti-CCP-positive RA was compared with non-RA patients (table 3) as opposed to when anti-CCP-negative RA was compared with non-RA patients (table 4).

To assess the performance of these antibodies in patients with longer disease duration, levels were measured in patients with established RA and persistent non-RA arthritis presenting with symptoms of $>3$ months' duration. Here IgG anti-CarVimentin and anti-AcVimentin antibodies showed 100\% specificity although sensitivities remained low (see online supplementary table S2). 
A a

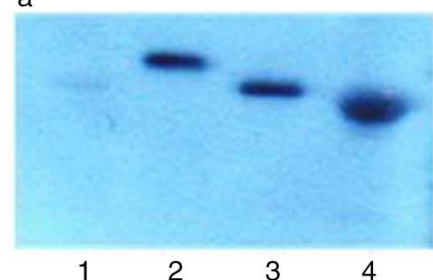

C

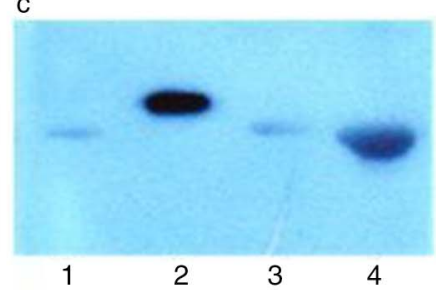

b

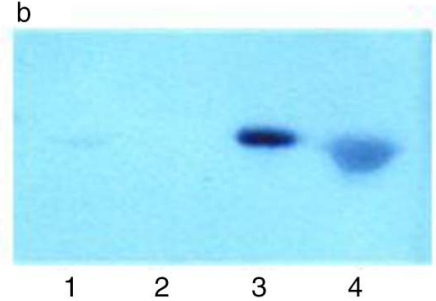

d

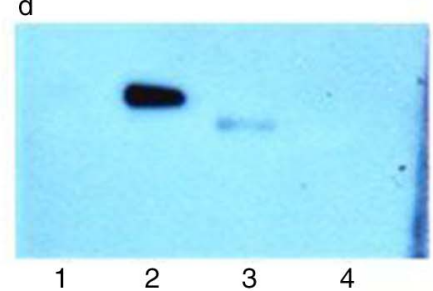

B

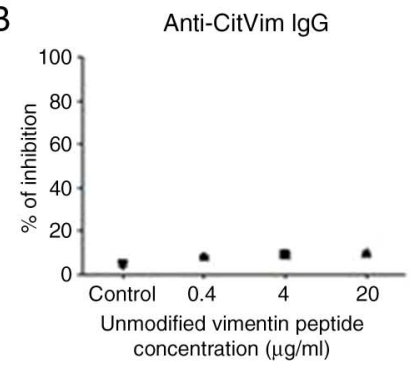

C

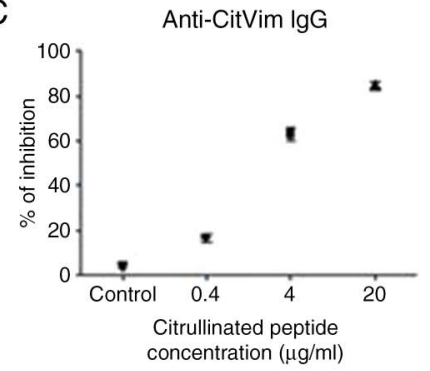

D

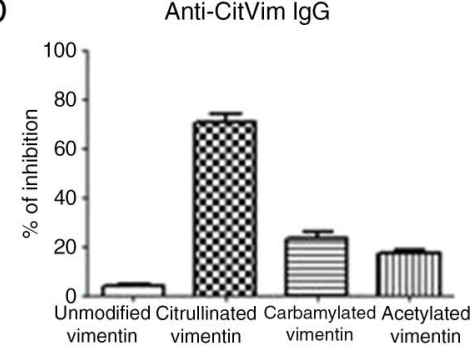

Anti-CarVim IgG

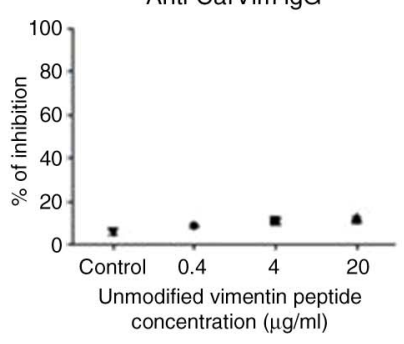

Anti-CarVim IgG

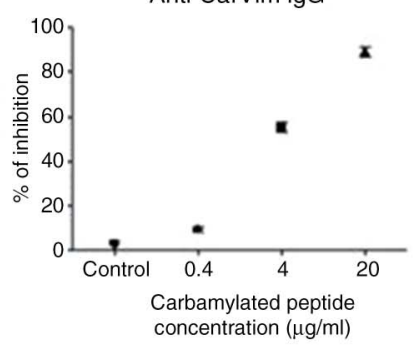

Anti-CarVim IgG

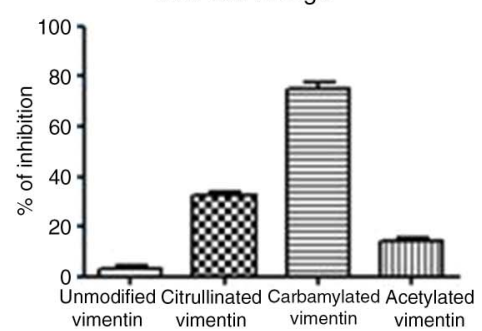

Anti-AcVim IgG

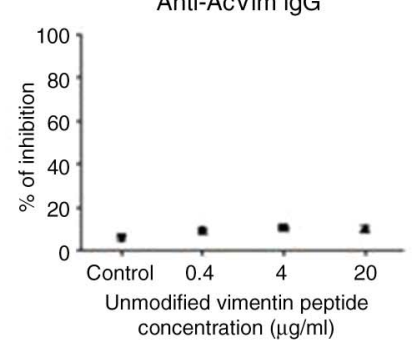

Anti-AcVim IgG

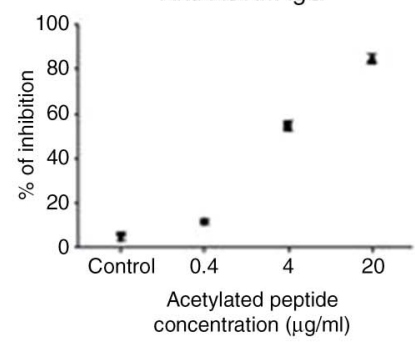

Anti-AcVim IgG

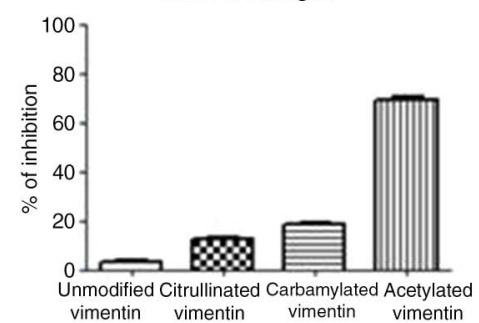

Figure 2 Competition studies. (A) Preincubation of post-translationally modified peptides with sera containing anticitrullinated, anticarbamylated and antiacetylated antibodies specifically blocks full-length modified vimentin protein binding. Representative western blot image shown. In each western blot, the following antigens were applied to the SDS-PAGE separation: lane 1, human recombinant vimentin; lane 2, citrullinated human recombinant vimentin; lane 3, carbamylated human recombinant vimentin; lane 4, acetylated vimentin-albumin conjugate. (A) Immunoblot with serum from a patients with rheumatoid arthritis (RA) cross-reacting with three different modified peptides. (B-D) Same experimental conditions as in (A), but the serum was preincubated with $10 \mu \mathrm{g} / \mathrm{mL}$ of citrullinated vimentin (B), carbamylated vimentin (C) or acetylated isoform (D) for $2 \mathrm{~h}$ prior to the immunoblotting experiment. Immunoassay competition experiments: (B) preincubation of patient sera with unmodified vimentin peptide did not inhibit IgG binding for any of the antibodies tested. Control denotes non-preincubated sera. (C) Preincubation of sera with corresponding modified peptides inhibited IgG binding in a dose-dependent manner. (D) Cross-reactivity between antibodies against different modifications. Binding of IgG antibodies to anti-CitVimentin peptides is inhibited by preincubation with citrullinated vimentin predominantly with low degree of inhibition by preincubation with carbamylated or acetylated vimentin. The same pattern is observed for anti-AcVimentin antibodies. Binding of anti-CarVimentin IgG antibodies is predominantly inhibited by preincubation with carbamylated vimentin but inhibition is also observed when sera is preincubated with citrullinated vimentin indicating a degree of cross-reactivity. Bars represent mean, error bars SD. CitVim, citrullinated vimentin peptide; CarVim, carbamylated vimentin peptide; AcVim, acetylated vimentin peptide. 
Table 2 Sensitivity, specificity, positive predictive value and negative predictive value of antibody isotypes when comparing patients with RA versus non-RA patients (persistent non-RA and resolving)

\begin{tabular}{|c|c|c|c|c|}
\hline & Sensitivity $(95 \% \mathrm{CI})$ & Specificity $(95 \% \mathrm{CI})$ & PPV $(95 \% \mathrm{Cl})$ & NPV $(95 \% \mathrm{Cl})$ \\
\hline Anti-CCP & $47.5 \%(37.5 \%$ to $57.7 \%)$ & $98.2 \%(94.8 \%$ to $99.6 \%)$ & $94.1 \%(83.7 \%$ to $98.7 \%)$ & $75.6 \%(69.3 \%$ to $81.1 \%)$ \\
\hline \multicolumn{5}{|c|}{ Rheum factor } \\
\hline $\lg G$ & $55.5 \%(45.2 \%$ to $65.3 \%)$ & $92.2 \%(87.0 \%$ to $95.7 \%)$ & $81.2 \%(69.9 \%$ to $89.6 \%)$ & $77.4 \%(70.9 \%$ to $83.0 \%)$ \\
\hline $\lg A$ & $33.6 \%(24.6 \%$ to $43.8 \%)$ & $91.0 \%(85.6 \%$ to $94.8 \%)$ & $69.4 \%(54.6 \%$ to $81.8 \%)$ & $69.4 \%(62.8 \%$ to $75.4 \%)$ \\
\hline \multicolumn{5}{|c|}{ Anti-CitVimentin } \\
\hline $\lg G$ & $56.4 \%(46.2 \%$ to $66.3 \%)$ & $83.2 \%(76.7 \%$ to $88.6 \%)$ & $67.1 \%(56.0 \%$ to $76.9 \%)$ & $76 \%(69.1 \%$ to $81.9 \%)$ \\
\hline $\lg A$ & $43.6 \%(33.7 \%$ to $53.8 \%)$ & $73.1 \%(65.6 \%$ to $79.6 \%)$ & $49.4 \%(38.7 \%$ to $60.3 \%)$ & $68.2 \%(60.8 \%$ to $74.9 \%)$ \\
\hline \multicolumn{5}{|c|}{ Anti-CarVimentin } \\
\hline $\lg G$ & $34.7 \%(25.5 \%$ to $44.8 \%)$ & $94 \%(89.3 \%$ to $97.1 \%)$ & $77.8 \%(62.9 \%$ to $88.9 \%)$ & $70.4 \%(63.9 \%$ to $76.3 \%)$ \\
\hline $\lg A$ & $16.8 \%(10.1 \%$ to $25.6 \%)$ & $88 \%(82.1 \%$ to $92.5 \%)$ & $45.9 \%(29.5 \%$ to $63.1 \%)$ & $63.6 \%(57.1 \%$ to $69.8 \%)$ \\
\hline \multicolumn{5}{|c|}{ Anti-AcVimentin } \\
\hline $\lg G$ & $36.6 \%(27.3 \%$ to $46.8 \%)$ & $86.2 \%(80.1 \%$ to $91.1 \%)$ & $61.7 \%(48.2 \%$ to $73.9 \%)$ & $69.2 \%(62.5 \%$ to $75.4 \%)$ \\
\hline $\lg A$ & $35.6 \%(26.4 \%$ to $45.8 \%)$ & $64.7 \%(56.9 \%$ to $71.9 \%)$ & $37.9 \%(28.2 \%$ to $48.4 \%)$ & $62.4 \%(54.8 \%$ to $69.7 \%)$ \\
\hline
\end{tabular}

Table 3 Sensitivity, specificity, positive predictive value and negative predictive value of antibody isotypes when comparing patients with anti-CCP-positive RA versus non-RA patients (persistent non-RA and resolving)

\begin{tabular}{|c|c|c|c|c|}
\hline & Sensitivity $(95 \% \mathrm{Cl})$ & Specificity $(95 \% \mathrm{Cl})$ & PPV (95\% Cl) & NPV (95\% Cl) \\
\hline Anti-CCP & $100 \%(92.5 \%$ to $100 \%)$ & $98.2 \%(94.8 \%$ to $99.6 \%)$ & $94.1 \%(83.7 \%$ to $98.7 \%)$ & $100 \%(97.8 \%$ to $100 \%)$ \\
\hline \multicolumn{5}{|c|}{ Rheum factor } \\
\hline $\lg G$ & $93.8 \%(82.8 \%$ to $98.6 \%)$ & $92.2 \%(87.1 \%$ to $95.8 \%)$ & $77.6 \%(64.7 \%$ to $87.5 \%)$ & $98.1 \%(94.5 \%$ to $99.6 \%)$ \\
\hline $\lg A$ & $56.3 \%(41.2 \%$ to $70.5 \%)$ & $91.0 \%(85.6 \%$ to $94.9 \%)$ & $64.3 \%(48.0 \%$ to $78.4 \%)$ & $87.9 \%(82.0 \%$ to $92.3 \%)$ \\
\hline \multicolumn{5}{|c|}{ Anti-CitVimentin } \\
\hline $\lg G$ & $93.8 \%(82.8 \%$ to $98.6 \%)$ & $83.3 \%(76.7 \%$ to $88.6 \%)$ & $61.6 \%(49.5 \%$ to $72.8 \%)$ & $97.9 \%(93.9 \%$ to $99.5 \%)$ \\
\hline $\lg A$ & $62.5 \%(47.5 \%$ to $76.0 \%)$ & $73.2 \%(65.6 \%$ to $79.6 \%)$ & $40.0 \%(28.9 \%$ to $51.9 \%)$ & $87.1 \%(80.4 \%$ to $92.2 \%)$ \\
\hline \multicolumn{5}{|c|}{ Anti-CarVimentin } \\
\hline $\lg G$ & $68.8 \%(53.8 \%$ to $81.3 \%)$ & $94.0 \%(89.3 \%$ to $97.1 \%)$ & $76.7 \%(61.4 \%$ to $88.2 \%)$ & $91.3 \%(86.0 \%$ to $95.0 \%)$ \\
\hline $\lg A$ & $16.7 \%(7.5 \%$ to $30.2 \%)$ & $88.0 \%(82.1 \%$ to $92.5 \%$ & $28.6 \%(13.3 \%$ to $48.7 \%)$ & $78.6 \%(72.0 \%$ to $84.3 \%)$ \\
\hline \multicolumn{5}{|c|}{ Anti-AcVimentin } \\
\hline $\lg G$ & $62.5 \%(47.5 \%$ to $76.0 \%)$ & $86.2 \%(80.1 \%$ to $91.1 \%)$ & $56.6 \%(42.3 \%$ to $70.2 \%)$ & $88.9 \%(83.0 \%$ to $93.3 \%)$ \\
\hline $\lg A$ & $41.7 \%(27.6 \%$ to $56.8 \%$ & $64.7 \%(56.9 \%$ to $71.9 \%)$ & $25.3 \%(16.2 \%$ to $36.4 \%)$ & $79.4 \%$ (71.6\% to $85.9 \%)$ \\
\hline
\end{tabular}

Table 4 Sensitivity, specificity, positive predictive value and negative predictive value of antibody isotypes when comparing patients with anti-CCP-negative RA versus non-RA patients (persistent non-RA and resolving)

\begin{tabular}{|c|c|c|c|c|}
\hline & Sensitivity $(95 \% \mathrm{Cl})$ & Specificity $(95 \% \mathrm{CI})$ & PPV $(95 \% \mathrm{CI})$ & NPV $(95 \% \mathrm{Cl})$ \\
\hline Anti-CCP & $0.0 \%(0.0 \%$ to $6.8 \%)$ & $98.2 \%(94.8 \%$ to $99.6 \%)$ & $0.0 \%(0.0 \%$ to $69.5 \%)$ & $75.6 \%(69.3 \%$ to $81.1 \%)$ \\
\hline \multicolumn{5}{|c|}{ Rheum factor } \\
\hline $\lg G$ & $20.8 \%(10.9 \%$ to $34.1 \%)$ & $92.2 \%(87.1 \%$ to $95.8 \%)$ & $45.8 \%(25.6 \%$ to $67.2 \%)$ & $78.6 \%(72.2 \%$ to $84.1 \%)$ \\
\hline $\lg A$ & $13.2 \%$ (5.5\% to $25.4 \%)$ & $91.0 \%(85.6 \%$ to $94.9 \%)$ & $31.8 \%(13.9 \%$ to $54.9 \%)$ & $76.8 \%(70.3 \%$ to $82.5 \%)$ \\
\hline \multicolumn{5}{|c|}{ Anti-CitVimentin } \\
\hline $\lg G$ & $22.6 \%((12.3 \%$ to $36.2 \%)$ & $83.2 \%$ (76.7\% to $88.6 \%)$ & $30.0 \%(16.6 \%$ to $46.5 \%)$ & $77.2 \%(70.4 \%$ to $83.2 \%)$ \\
\hline $\lg A$ & $26.4 \%(15.3 \%$ to $40.3 \%)$ & $73.1 \%(65.6 \%$ to $79.6 \%)$ & $23.7 \%(13.6 \%$ to $36.6 \%)$ & $75.8 \%(68.4 \%$ to $82.2 \%)$ \\
\hline \multicolumn{5}{|c|}{ Anti-CarVimentin } \\
\hline $\lg G$ & $3.8 \%(0.6 \%$ to $13.0 \%)$ & $94.0 \%(89.3 \%$ to $97.1 \%)$ & $16.7 \%$ (2.6\% to $48.4 \%)$ & $75.5 \%(69.1 \%$ to $81.2 \%)$ \\
\hline $\lg A$ & $16.9 \%(8.1 \%$ to $29.8 \%)$ & $88.0 \%(82.1 \%$ to $92.5 \%)$ & $31.0 \%(15.3 \%$ to $50.8 \%)$ & $76.9 \%(70.3 \%$ to $82.7 \%)$ \\
\hline \multicolumn{5}{|c|}{ Anti-AcVimentin } \\
\hline $\lg G$ & $13.2 \%(5.5 \%$ to $25.4 \%)$ & $86.2 \%(80.1 \%$ to $91.1 \%)$ & $23.3 \%$ (9.9\% to $42.3 \%)$ & $75.8 \%(69.1 \%$ to $81.7 \%)$ \\
\hline $\lg A$ & $30.2 \%(18.4 \%$ to $44.3 \%)$ & $64.7 \%$ (56.9\% to $71.9 \%)$ & $21.3 \%(12.7 \%$ to $32.3 \%)$ & $74.5 \%$ (66.6\% to $81.4 \%)$ \\
\hline
\end{tabular}

AcVim, acetylated vimentin peptide; CarVim, carbamylated vimentin peptide; CitVim, citrullinated vimentin peptide; NPV, negative predictive value; PPV, positive predictive value; RA, rheumatoid arthritis. 


\section{Comparison of antibody profiles between outcome groups}

To investigate the individual antibody profiles, the RA group was subcategorised into anti-CCP-positive and anti-CCPnegative as defined by the anti-CCP2 assay. The frequency of antibody seropositivity in the four outcome groups is represented in figure $3 \mathrm{~A}, \mathrm{~B}$.

To further investigate specific antibody reactivities, the number of peptides recognised by patients in each outcome group was determined. For IgG antibodies, there were clear differences in the number of peptides recognised between patients with anti-CCP-positive RA and the remaining groups (figure 3C). While $58.3 \%$ of anti-CCP-positive patients had antibodies recognising all three peptides, only small proportions of patients in the anti-CCP-negative RA, persistent non-RA and resolving groups $(1.9 \%, 5.5 \%$ and $5.4 \%$, respectively, $\mathrm{p}<0.0001)$ recognised all three peptides. Conversely, while $73-80 \%$ of patients in the anti-CCP-negative, persistent non-RA and resolving groups showed no reactivity to any of the peptides, only $6.3 \%$ of anti-CCP-positive patients tested negative for antibodies against these peptides $(\mathrm{p}<0.0001)$. A graphical representation of the individual IgG antibody profiles of patients in all outcome groups is shown in figure 4.

\section{Characteristics of patients recognising all three peptides containing post-translational modifications}

The demographic and clinical characteristics of patients whose sera tested positive for IgG antibodies against all three PTMP were compared with the remaining patients. Anti-CCP and RF status were the only significant differences between groups $(p<0.0001$ for both). Logistic regression demonstrated that patients with anti-CCP-positive RA were more likely to have positive anti-CitVimentin (OR 60, 95\% CI 19.6 to $183.5, \mathrm{p}<0.0001$ ), anti-CarVimentin (OR 33, 95\% CI 14.1 to 77.1, p<0.0001) and anti-AcVimentin (OR 10.6, 95\% CI 5.2 to $21.8, \mathrm{p}<0.0001$ ) antibodies than patients with anti-CCP-negative RA.

\section{DISCUSSION}

We show that a range of $\operatorname{IgG}$ and IgA antibodies against vimentin-derived PTMP are present in the sera of patients with early inflammatory arthritis. In the context of IgG antibodies, two distinct patterns arise, one for patients with anti-CCPpositive RA and another for the remaining diagnostic categories. Negligible reactivity to the unmodified control peptide was seen across all groups, indicating that PTM of peptide is needed for reactivity.

This is the first description of anti-AcVimentin antibodies in the sera of patients with early RA. Lysine acetylation is a dynamic process that requires a tight balance between acetylases and deacetylases to maintain appropriate acetylation profiles required for normal cellular function. ${ }^{22}$ Acetylation profiles are altered in a variety of diseases and can be modulated by certain dietary components in vivo. ${ }^{23}$ The microbiome has recently also been shown to affect acetylation patterns in vivo. ${ }^{18}$ De novo acetylation of lysine residues in hepatic and colonic proteins was seen following colonisation of germ-free raised mice with microbiota from conventionally raised mice. ${ }^{18}$ The observation that certain gut bacteria influence host cells lysine acetylation is of potential relevance to RA. Infections have long been linked to RA; the disease-modifying drug sulfasalazine has an antibiotic effect, and the antibiotic doxycycline has disease-modifying effects. ${ }^{23}$ Disease is attenuated in arthritis animal models if they are raised in germ-free conditions or treated with antibiotics while introduction of specific gut bacteria induces joint inflammation. ${ }^{24} 25$ However, the mechanisms
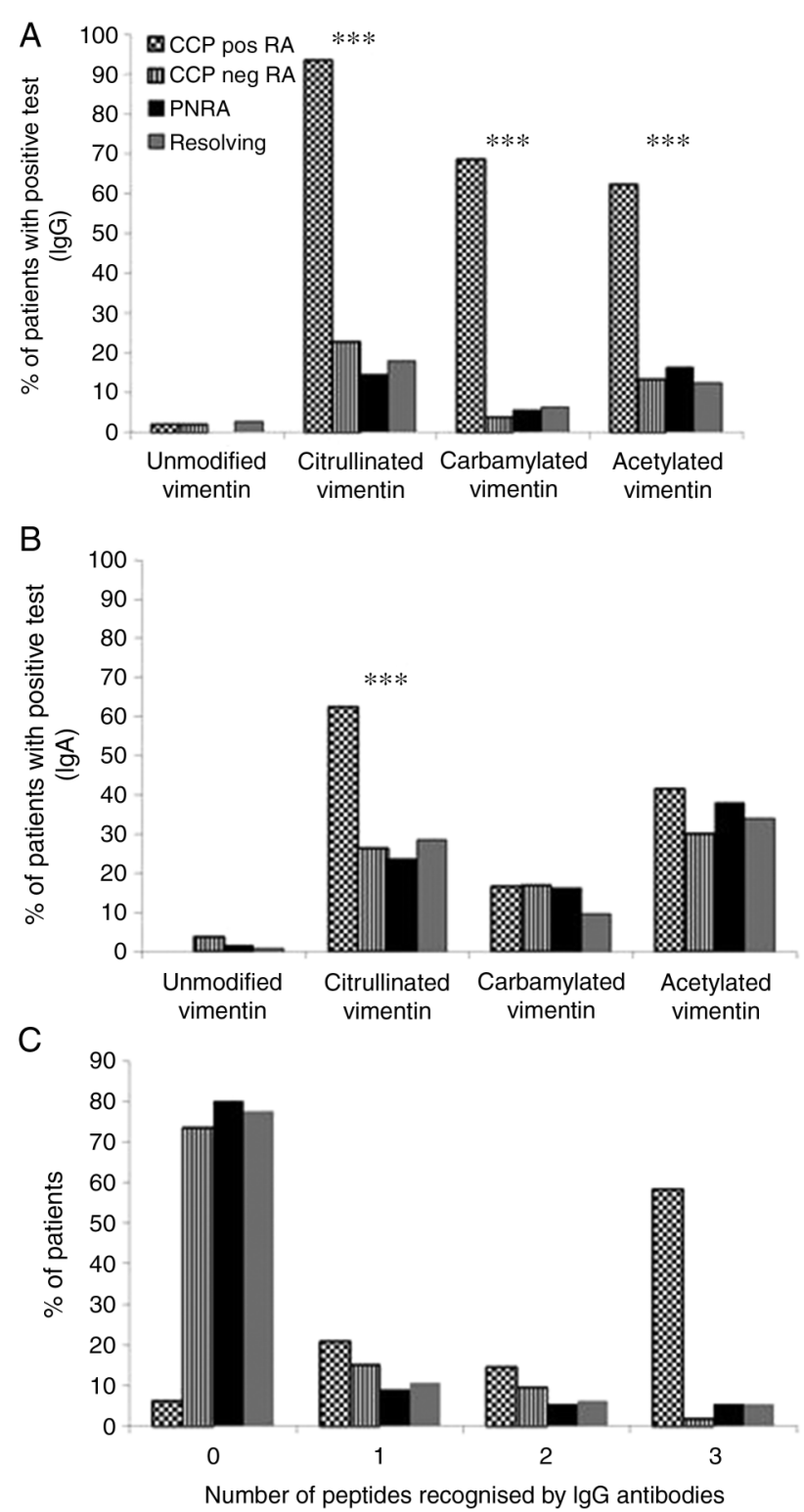

Figure 3 Frequency of antibody positivity to different posttranslationally modified peptides in each outcome group. Anti cyclic citrullinated peptide (CCP)-positive rheumatoid arthritis (RA) (checkered bar), anti-CCP-negative RA (vertical lines bar), persistent non-RA arthritis (black bar) and resolving arthritis (grey bar). (A) IgG reactivities: statistically significant differences in the percentages of patients with positive antibodies were observed between patients with anti-CCP-positive RA and the remaining groups for all peptides. (B) $\lg A$ reactivities: statistically significant differences in the frequency of anti-CitVimentin IgA antibody reactivities seen between patients with anti-CCP-positive RA and the remaining groups but not for the remaining post-translationally modified peptides. ${ }^{* *} p \leq 0.001$. (C) Distribution of the number of peptides recognised according to outcome group. The highest proportion of patients with anti-CCP-positive RA recognised three peptides while the highest proportions of patients in the remaining outcome groups did not recognise any peptides.

explaining these effects remain undefined. Recently, Scher $e t a l^{26}$ identified evidence of Prevotella copri in faeces from $75 \%$ of new onset treatment-naive patients with RA, a significantly higher percentage than found in patients with long-standing treated RA, treated psoriatic arthritis and healthy controls. The significance of our finding of anti-AcVimentin antibodies in the sera of patients with anti-CCP-positive RA will need to be elucidated. However, it 

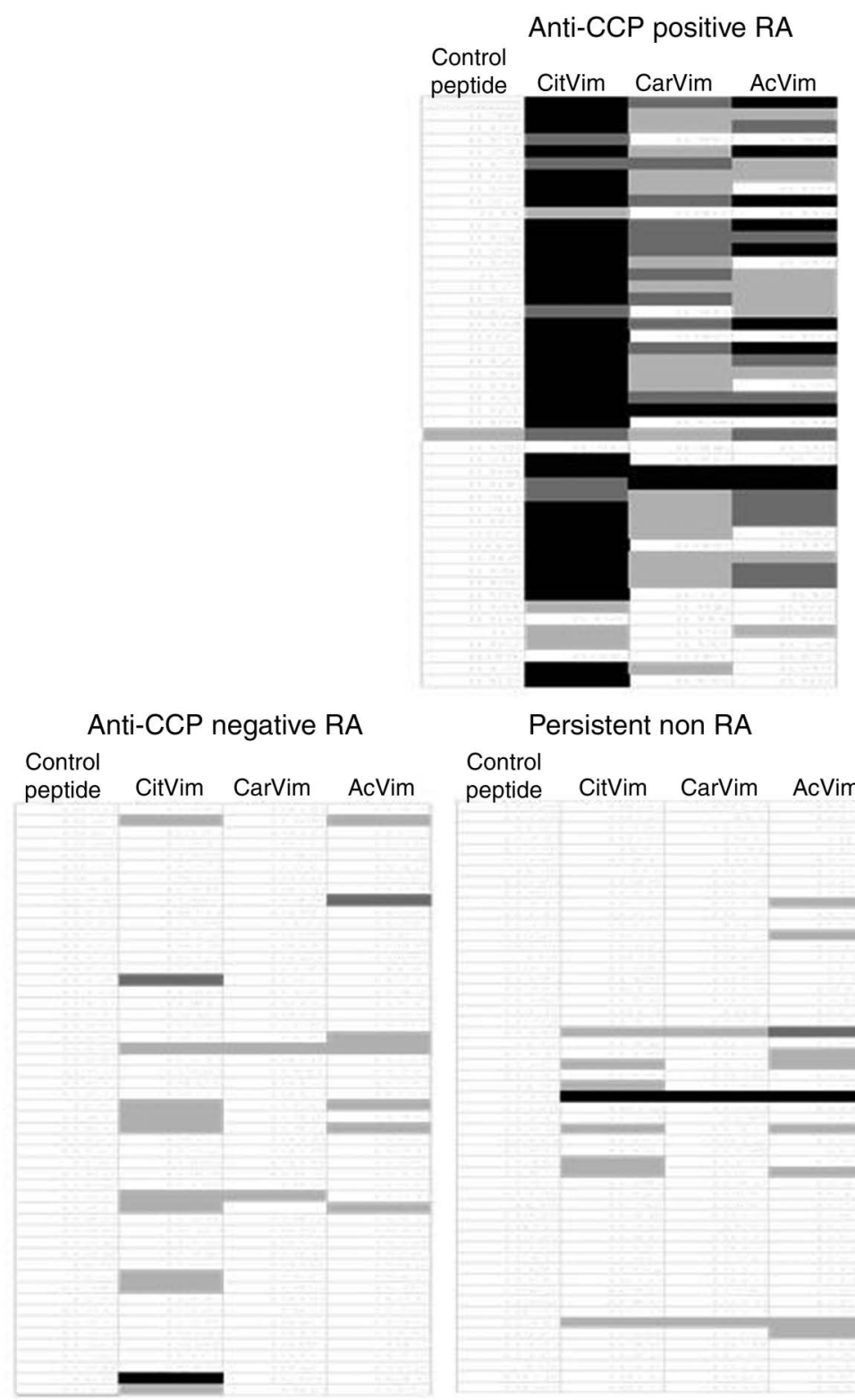

Persistent non RA

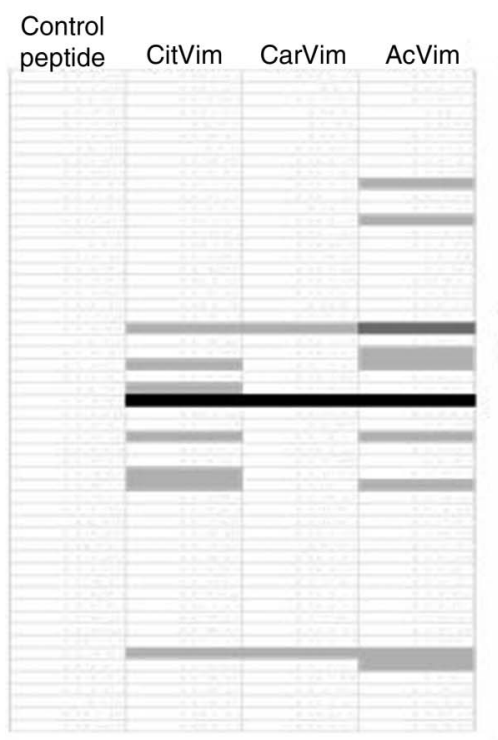

Resolving arthritis

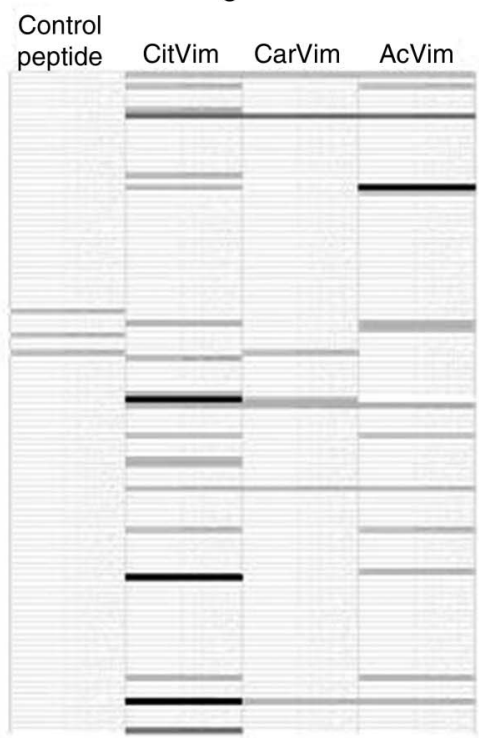

Figure 4 Graphical representation of IgG antibody reactivity results. Patients with anti cyclic citrullinated peptide (CCP)-positive rheumatoid arthritis (RA) showed a distinct antibody profile that differed from that of the remaining outcome groups. This patient group had reactivities against a higher number of peptides and the antibody levels against those peptides were also higher. Shaded squares represent positive tests: light grey, low-level positivity (OD >98th percentile to 1.0); dark grey, medium positivity (OD >1.0-1.9); black, high positivity (OD >1.9). Each row represents an individual patient, each column represents a peptide. Control, native unmodified vimentin peptide; CitVim, citrullinated vimentin peptide; CarVim, carbamylated vimentin peptide; AcVim, acetylated vimentin peptide.

is possible that there may be a link between the microbiome, selfantigen acetylation and autoimmunity. The test characteristics of anti-AcVimentin antibodies are relatively poor for predicting the development of anti-CCP-negative RA, suggesting that the identification of these antibodies is unlikely to be useful in this context. Future work will need to address their role in predicting disease severity and other clinically relevant outcomes in patients with RA.

Anticarbamylated protein antibodies have been identified in the sera of patients with early RA of $<2$ years' duration. ${ }^{8}$ Antibodies were present in $37 \%$ of ACPA-positive and $16 \%$ of ACPA-negative patients. ${ }^{8}$ In a subsequent study from the same group, anticarbamylated antibody positivity was assessed in two large RA cohorts and mainly detected in the anti-CCP-positive group, more consistent with our results. ${ }^{27}$ We show that $68.7 \%$ of anti-CCP-positive and 3\% of anti-CCP-negative patients with RA have anti-CarVimentin antibodies. Differing frequencies between studies may be explained by differing study designs, sample sizes, disease durations and/or differences in antigens used. While the antigen we used was a vimentin-derived peptide of known sequence where homocitrulline was introduced at a specific position, Shi et al used carbamylated fetal calf serum (FCS). FCS contains multiple proteins, and a number of different amino acids may be modified during the carbamylation reaction, resulting in multiple potential epitopes. Furthermore, carbamylation reactions require very specific reaction conditions. Factors such as temperature, amount of cyanate used and reaction duration will determine the number of lysine residues that are carbamylated. Consequently, this may result in 
generation of differing antigen epitopes in different reactions. In contrast, by introducing homocitrulline in a specific position of the peptide, we ensured that reactivity against a specific known antigen was consistently tested.

While cross-reactivity between anticarbamylated protein antibodies and ACPA is estimated at $30 \%,{ }^{28}$ no association between anticarbamylated antibody positivity and smoking or human leucocyte antigen (HLA)-DRB alleles has been found, suggesting that mechanisms underlying anticarbamylated antibody and ACPA formation differ. ${ }^{27}$ We show a degree of cross-reactivity between antibody families that is comparable to that of published work. The existence of cross-reactive antibodies was also suggested by the first characterisation of anticarbamylated antibodies where immunisation of rabbits with homocitrullinated human albumin triggered anti-CCP2 and anti-MCV antibody generation. ${ }^{10}$ Cross-reactivity between anticarbamylated fibrinogen antibodies and ACPA in the sera of patients with RA has recently been described. ${ }^{29}$ The fact that antibodies do not bind single amino acids but rather a structural epitope formed of many amino acids must be considered in this context. The majority of cross-reactive antibodies likely occur because protein regions that contain homocitrulline structurally resemble an alternative protein region that contains citrulline.

A strength of our study is that the design of specific peptides in which each modification is introduced sequentially, together with the results of the competition experiments, enabled us to distinguish specific antibody reactivities. Inhibition experiments showed limited cross-reactivity between antiacetylated, anticarbamylated and anticitrullinated vimentin peptide antibodies, indicating that these are different antibody families. While citrullinated antigens other than vimentin (eg, fibrinogen) generate ACPA reactivity, for this work we chose a vimentin-derived peptide as antigen. We reasoned that to maximise the yield of novel autoantibodies identified, PTMs should be introduced on the backbone of an already well-known autoantibody trigger. This backbone was derived from the protein that is used in the MCV assay (of known high sensitivity and specificity) and that is present in RA joints. ${ }^{7}$ Furthermore, an epitope present in the peptide we used is preferentially recognised by $\mathrm{ACPA}^{4}$ and HLA-DRB1 binds with high affinity to citrullinated vimentin peptides generating $\mathrm{T}$ cell response, ${ }^{30}$ all of which led us to use this as our candidate peptide backbone. Now that the presence of antiacetylated antibodies has been established, reactivity against other acetylated peptides and proteins should be assessed to determine whether higher sensitivity and specificity to other acetylated substrates exists. Future work should also be directed towards identification of acetylated proteins in diseased RA joints.

In summary, these data suggest that, like citrullination, carbamylation and acetylation may be involved in the pathogenesis of RA by triggering the generation of autoantibodies and/or by generating targets for antibody responses in the rheumatoid joint.

Contributors $K R, H B, C D B$ and $A F$ designed the study. KR, MJ, BD, IS, BF and $A F$ contributed to patient recruitment to the BEACON cohort. $H B, F H$ and UR performed and/or supervised laboratory experiments to measure anti-PTMP antibodies and validate the laboratory findings. $M J, K R, A F, B F, B D, I S$ and $H B$ were involved in data analysis. All authors were involved in data interpretation and writing of the manuscript.

Funding The research leading to these results was funded within the FP7 HEALTH programme under the grant agreement FP7-HEALTH-F2-2012-305549.

Competing interests None declared.

Patient consent Obtained.
Ethics approval Solihull Local Ethics Research Committee.

Provenance and peer review Not commissioned; externally peer reviewed.

Open Access This is an Open Access article distributed in accordance with the Creative Commons Attribution Non Commercial (CC BY-NC 4.0) license, which permits others to distribute, remix, adapt, build upon this work non-commercially, and license their derivative works on different terms, provided the original work is properly cited and the use is non-commercial. See: http://creativecommons.org/ licenses/by-nc/4.0/

\section{REFERENCES}

1 Gerlag DM, Raza K, van Baarsen LG, et al. EULAR recommendations for terminology and research in individuals at risk of rheumatoid arthritis: report from the Study Group for Risk Factors for Rheumatoid Arthritis. Ann Rheum Dis 2012;71:638-41.

2 Willemze A, Toes RE, Huizinga TW, et al. New biomarkers in rheumatoid arthritis. Neth J Med 2012;70:392-9.

3 Raza K, Breese M, Nightingale $P$, et al. Predictive value of antibodies to cyclic citrullinated peptide in patients with very early inflammatory arthritis. J Rheumatol 2005:32:231-8.

4 Harre U, Georgess D, Bang H, et al. Induction of osteoclastogenesis and bone loss by human autoantibodies against citrullinated vimentin. J Clin Invest 2012;122:1791-802.

5 Aggarwal R, Liao K, Nair R, et al. Anti-citrullinated peptide antibody assays and their role in the diagnosis of rheumatoid arthritis. Arthritis Rheum 2009;61:1472-83.

6 Wegner N, Lundberg K, Kinloch A, et al. Autoimmunity to specific citrullinated proteins gives the first clues to the etiology of rheumatoid arthritis. Immunol Rev 2010;233:34-54.

7 Bang $H$, Egerer $K$, Gauliard A, et al. Mutation and citrullination modifies vimentin to a novel autoantigen for rheumatoid arthritis. Arthritis Rheum 2007;56:2503-11.

8 Shi J, Knevel R, Suwannalai $P$, et al. Autoantibodies recognizing carbamylated proteins are present in sera of patients with rheumatoid arthritis and predict joint damage. Proc Natl Acad Sci USA 2011;108:17372-7.

9 Gyorgy B, Toth E, Tarcsa E, et al. Citrullination: a posttranslational modification in health and disease. Int J Biochem Cell Biol 2006;38:1662-77.

10 Turunen S, Koivula MK, Risteli L, et al. Anticitrulline antibodies can be caused by homocitrulline-containing proteins in rabbits. Arthritis Rheum 2010;62:3345-52.

11 Jaisson S, Gillery P. Evaluation of nonenzymatic posttranslational modification-derived products as biomarkers of molecular aging of proteins. Clin Chem 2010;56:1401-12.

12 Makrygiannakis D, Hermansson M, Ulfgren AK, et al. Smoking increases peptidylarginine deiminase 2 enzyme expression in human lungs and increases citrullination in BAL cells. Ann Rheum Dis 2008;67:1488-92.

13 Reynisdottir G, Karimi R, Joshua V, et al. Structural changes and antibody enrichment in the lungs are early features of anti-citrullinated protein antibody-positive rheumatoid arthritis. Arthritis Rheumatol 2014;66:31-9.

14 Klareskog L, Stolt P, Lundberg K, et al. A new model for an etiology of rheumatoid arthritis: smoking may trigger HLA-DR (shared epitope)-restricted immune reactions to autoantigens modified by citrullination. Arthritis Rheum 2006;54:38-46.

15 Yang XJ, Seto E. Lysine acetylation: codified crosstalk with other posttranslational modifications. Mol Cell 2008;31:449-61.

16 Kim GW, Yang XJ. Comprehensive lysine acetylomes emerging from bacteria to humans. Trends Biochem Sci 2011;36:211-20.

17 Zhao $S, X u$ W, Jiang $W$, et al. Regulation of cellular metabolism by protein lysine acetylation. Science 2010;327:1000-4.

18 Simon GM, Cheng J, Gordon Jl. Quantitative assessment of the impact of the gut microbiota on lysine epsilon-acetylation of host proteins using gnotobiotic mice. Proc Natl Acad Sci USA 2012;109:11133-8.

19 Arnett FC, Edworthy SM, Bloch DA, et al. The American Rheumatism Association 1987 revised criteria for the classification of rheumatoid arthritis. Arthritis Rheum 1988:31:315-24.

20 Aletaha D, Neogi T, Silman AJ, et al. 2010 rheumatoid arthritis classification criteria: an American College of Rheumatology/European League Against Rheumatism collaborative initiative. Ann Rheum Dis 2010;69:1580-8.

21 Ramponin G, Manao G, Camici G. Nonenzymatic acetylation of histones by acetyl phosphate and acetyl adenylate. Biochemistry 1975;14:2681-5.

22 Kim GW, Gocevski G, Wu CJ, et al. Dietary, Metabolic, and Potentially Environmental Modulation of the Lysine Acetylation Machinery. Int J Cell Biol 2010;2010:632739.

23 O'Dell JR, Elliott JR, Mallek JA, et al. Treatment of early seropositive rheumatoid arthritis: doxycycline plus methotrexate versus methotrexate alone. Arthritis Rheum 2006;54:621-7.

24 Rath HC, Herfarth HH, Ikeda JS, et al. Normal luminal bacteria, especially Bacteroides species, mediate chronic colitis, gastritis, and arthritis in HLA-B27/human beta2 microglobulin transgenic rats. J Clin Invest 1996; 98:945-53. 
25 Horai R, Saijo S, Tanioka $H$, et al. Development of chronic inflammatory arthropathy resembling rheumatoid arthritis in interleukin 1 receptor antagonist-deficient mice. $J$ Exp Med 2000;191:313-20.

26 Scher JU, Sczesnak A, Longman RS, et al. Expansion of intestinal Prevotella copri correlates with enhanced susceptibility to arthritis. Elife 2013;2:e01202.

27 Jiang $X$, Trouw LA, van Wesemael TJ, et al. Anti-CarP antibodies in two large cohorts of patients with rheumatoid arthritis and their relationship to genetic risk factors, cigarette smoking and other autoantibodies. Ann Rheum Dis 2014; $73: 1761-8$
28 Shi J, Willemze A, Janssen GM, et al. Recognition of citrullinated and carbamylated proteins by human antibodies: specificity, cross-reactivity and the "AMC-Sneshu" method. Ann Rheum Dis 2013;72:148-50.

29 Scinocca M, Bell DA, Racape $M$, et al. Antihomocitrullinated fibrinogen antibodies are specific to rheumatoid arthritis and frequently bind citrullinated proteins/ peptides. J Rheumatol 2014;41:270-9.

30 Hill JA, Southwood S, Sette A, et al. Cutting edge: the conversion of arginine to citrulline allows for a high-affinity peptide interaction with the rheumatoid arthritis associated HLA-DRB1*0401 MHC class II molecule. J Immunol 2003;171:538-41. 